TRANSACTIONS OF THE

AMERICAN MATHEMATICAL SOCIETY

Volume 358, Number 3, Pages 1187-1201

S 0002-9947(05)03752-9

Article electronically published on April 22, 2005

\title{
AUTOMORPHISMS OF FIBER SURFACES OF GENUS 2, INDUCING THE IDENTITY IN COHOMOLOGY
}

\author{
JIN-XING CAI
}

\begin{abstract}
Let $S$ be a complex non-singular projective surface of general type with a genus 2 fibration and $\chi\left(\mathcal{O}_{S}\right) \geq 5$. Let $G \subset$ Aut $S$ be a non-trivial subgroup of automorphisms of $S$, inducing trivial actions on $H^{i}(S, \mathbb{Q})$ for all $i$. Then $|G|=2, K_{S}^{2}=4 \chi\left(\mathcal{O}_{S}\right)$ and $q(S)=1$. Examples of such surfaces are given.
\end{abstract}

\section{INTRODUCTION}

It is well known that, for a curve $C$ of genus $g \geq 2$, the automorphism group Aut $C$ acts faithfully on $H^{1}(C, \mathbb{Q})$. The case of surfaces has been studied by many authors. For $\mathrm{K} 3$ and Enriques surfaces $S$, Aut $S$ acts faithfully on $H^{2}(S, \mathbb{Z})$ (cf. [BR, [Ue]); and there exists an Enriques surface $S$ for which Aut $S$ does not act faithfully on $H^{2}(S, \mathbb{Q})$ (cf. Pet]). Let $S$ be a complex non-singular projective surface of general type, and let $G \subset$ Aut $S$ be a non-trivial subgroup of automorphisms of $S$, inducing trivial actions on $H^{2}(S, \mathbb{Q})$. As shown by Peters Pet, if the canonical linear system $\left|K_{S}\right|$ is base point free, then either $K_{S}^{2}=8 \chi\left(\mathcal{O}_{S}\right)$ or $K_{S}^{2}=9 \chi\left(\mathcal{O}_{S}\right)$. Recently, we proved that $|G| \leq 4$ if $\chi\left(\mathcal{O}_{S}\right)>188$ [Ca2]. An interesting question is whether such pairs $(S, G)$ do really occur except for the well-known examples (the product of two hyperelliptic curves). In this note, we consider the case when $S$ has a fibration of genus 2 .

Theorem 1.1 (Proposition 3.1 and Theorem 3.2). Let $S$ be a complex non-singular projective surface of general type with a genus 2 fibration and $\chi\left(\mathcal{O}_{S}\right) \geq 5$. Let $G \subset \operatorname{Aut} S$ be a non-trivial subgroup of automorphisms of $S$, inducing trivial actions on $H^{i}(S, \mathbb{Q})$ for all $i$. Then

(i) $|G|=2$, and the generator $\sigma$ of $G$ is a bi-elliptic involution of $f$.

(ii) The canonical map of $S$ factors through $f$ and $S$ has the following numerical invariants:

$$
K_{S}^{2}=4 \chi\left(\mathcal{O}_{S}\right), \quad q(S)=g(C)=1 .
$$

Remark 1.2. The possible pairs $(S, G)$ given in Theorem 1.1 indeed exist (Example 3.3). It will be interesting to classify all the pairs of Theorem 1.1

Theorem 1.1 is proved in Sections $3-4$. We reduce the problem to the analysis of the possible configurations for singular fibers of $f$ and the action of $G$ on them.

We work over the complex number field and use the standard notations as in BPV.

Received by the editors October 31, 2003 and, in revised form, April 26, 2004.

2000 Mathematics Subject Classification. Primary 14J50; Secondary 14J29.

(C)2005 American Mathematical Society Reverts to public domain 28 years from publication 


\section{Preliminaries}

Let $S$ be a smooth complex projective surface, with a relatively minimal fibration $f: S \rightarrow C$ of genus $g \geq 2$ over a smooth curve $C$. An irreducible curve $D$ on $S$ is vertical (with respect to $f$ ) if $f(D)$ is a point; otherwise, we say $D$ is horizontal.

Let $\sigma \in$ Aut $S$. A curve $D \subset S$ is $\sigma$-invariant (resp. $\sigma$-fixed), if $\sigma(D)=D$ (resp. $\sigma(p)=p$ for any $p \in D)$.

2.1. If a reduced $\sigma$-fixed curve $D$ is singular, then $\sigma$ is trivial. This follows since the induced action of $\sigma$ on the tangent space at the singular point of $D$ is trivial.

2.2. Let $D$ be a smooth curve, and $G \subset \operatorname{Aut} D$ a finite group. If $G$ has a fixed point, then $G$ is cyclic.

2.3 (cf. [Pet, Lemma 2]). Let $G \subset$ Aut $S$ be a subgroup of automorphisms of $S$ inducing trivial actions on $H^{2}(S, \mathbb{Q})$. Assume $p_{g}(S)>0$. If an irreducible curve $D$ is $\sigma$-fixed for some id $\neq \sigma \in G$, then $D \subset \operatorname{Bs}\left|K_{S}\right|$, where $K_{S}$ is the canonical divisor of $S$.

2.4. Let $f: S \rightarrow C$ be as above, and let $\lambda$ be an involution of $S$ inducing the trivial action on $C$. Let $\rho: \tilde{S} \rightarrow S$ be the blowup of all isolated fixed points of $\lambda$, and let $\tilde{\lambda}$ be the induced involution on $\tilde{S}$. Then $f$ induces a fibration $h: \tilde{S} / \tilde{\lambda} \rightarrow C$ of genus $g(F / \lambda)$ (not relatively minimal in general). We have a commutative diagram

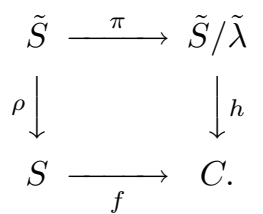

If $\Gamma<f^{*} c(c \in C)$ is a $\lambda$-fixed curve, then from $(f \circ \rho)^{*} c=\pi^{*}\left(h^{*} c\right)$, the coefficient of $\Gamma$ in $f^{*} c$ is divisible by two. In particular, if $f^{*} c$ is reduced, then $\lambda$ acts non-trivially on any irreducible component of $f^{*} c$.

2.5. Let $F^{\prime}$ be a singular fiber of $f$. An effective divisor $\Gamma<F^{\prime}$ is said to be an elliptic tail, if $\Gamma$ is 2-connected (cf. [BPV, p. 69]), and $K_{S} \Gamma=1, \Gamma^{2}=-1$. The configurations for elliptic tails $\Gamma$ are as follows:

(i) $\Gamma$ is either a smooth elliptic curve or a rational curve with a single node or cusp.

(ii) $\Gamma=\Gamma_{1}+\Theta$, where $\Gamma_{1}$ is -3 -curve and $\Theta$ a -2-cycle of type $A_{n}(n \geq 1)$. $\Gamma_{1}$ and $\Theta$ meet transversally in two points.

(iii) $\Gamma=\Gamma_{1}+\Theta$ with $\Gamma_{1} \Theta=2$, where $\Gamma_{1}$ is a -3 -curve, and $\Theta$ is either a -2-curve or a -2-cycle of type $D_{n}(n \geq 4)$ or $E_{n}(n=6,7,8) . \Gamma_{1}$ and $\Theta$ meet in one point.

2.6 (cf. [H] Lemma 8], [X1, p. 9]). Let $f: S \rightarrow C$ be a minimal fibration of genus 2 , and let $\tau$ be the hyperelliptic involution of $f$. Let $F^{\prime}$ be a singular fiber of $f$. If $F^{\prime}$ is not 2-connected, then it has one of the following configurations:

$\left(a_{k}\right)$ (type I and II in the sense of Horikawa) $F^{\prime}=\Gamma_{1}+\Theta_{1}+\cdots+\Theta_{k}+\Gamma_{2}$ :

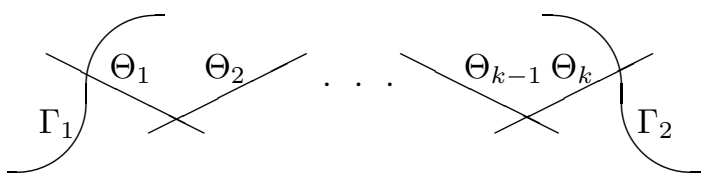


$\left(b_{k}\right)$ (type III, IV and V in the sense of Horikawa) $F^{\prime}=\Theta+\Theta^{\prime}+2 \Theta_{1}+2 \Theta_{2}+$ $\cdots+2 \Theta_{k}+2 \Gamma$ :

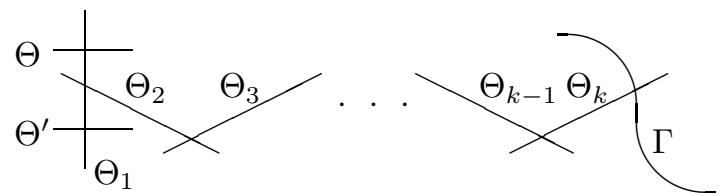

where $k \geq 0, \Gamma_{1}, \Gamma_{2}, \Gamma$ are elliptic tails, and $\Theta, \Theta^{\prime}, \Theta_{i}$ are -2 -curves.

2.7. Let $F^{\prime}$ be as in $\left(b_{k}\right)$ of 2.6. Then $F^{\prime}$ is of type III-IV or $\mathrm{V}$ depending on whether $\tau(\Theta)=\Theta^{\prime}$ or not. Moreover, if $F^{\prime}$ is of type $\mathrm{V}$, then $k \geq 1$.

2.8. If $F^{\prime}$ is as in $\left(a_{k}\right)$ of [2.6, then $\Gamma_{1} \cap \Theta_{1}, \Theta_{i} \cap \Theta_{i+1}(i=1, \cdots, k-1)$ and $\Theta_{k} \cap \Gamma_{2}$ are isolated $\tau$-fixed points. If $F^{\prime}$ is as in $\left(b_{k}\right)$ of 2.6 and $F^{\prime}$ is not of type $\mathrm{V}$, then $\Theta_{i} \cap \Theta_{i+1}(i=1, \cdots, k-1)$ and $\Theta_{k} \cap \Gamma$ are isolated $\tau$-fixed points, and there is precisely an isolated $\tau$-fixed point on $\Gamma$ when $k=0$ (resp. $\Theta_{1} \backslash \Theta_{1} \cap \Theta_{2}$ when $k>0)$.

2.9 (cf. e.g., X1, p. 13]). Let $f: S \rightarrow C$ be as in 2.9, The vertical part of the fixed part of $\left|K_{S}\right|$ is equal to $\sum_{\text {singular fiber } F^{\prime}} \Delta_{F^{\prime}}$, where $\Delta_{F^{\prime}}=0$ (resp. $\Theta_{1}+2 \Theta_{2}+$ $\left.3 \Theta_{3}+\cdots+2 \Theta_{k-1}+\Theta_{k},[(k+1) / 2]\left(\Theta+\Theta^{\prime}\right)+k \Theta_{1}+(k-1) \Theta_{2}+\cdots+2 \Theta_{k-1}+\Theta_{k}\right)$ if $F^{\prime}$ is either 2-connected or of type $\mathrm{V}$ (resp. of type $\left(a_{k}\right)$ of 2.6, of type III or IV as in $\left(b_{k}\right)$ of 2.6 ).

For more detail results on genus 2 fibration, we refer to $[\mathrm{H}, \mathrm{X} 1$ ].

\section{Reductions And Examples}

Proposition 3.1. Let $S$ be a complex non-singular projective surface of general type with $\chi\left(\mathcal{O}_{S}\right) \geq 5$, and let $f: S \rightarrow C$ be a relatively minimal fibration of genus 2. Let $G \subset$ Aut $S$ be a subgroup of automorphisms of $S$, inducing trivial actions on $H^{i}(S, \mathbb{Q})$ for all $i$. Assume that the canonical map $\phi_{S}$ of $S$ is generically finite. Then $G$ is trivial.

Proof. Suppose that $G$ is not trivial. Since $H^{0}\left(S, \omega_{S}\right)$ is a direct factor of $H^{2}(S, \mathbb{C})$, $G$ acts trivially on $H^{0}\left(S, \omega_{S}\right)$. This implies that $G$ induces trivial actions on $\operatorname{Im} \phi_{S}$. So $\phi_{S}$ factors through the quotient map

$$
\phi_{S}=\alpha \circ q: S \stackrel{q}{\longrightarrow} S / G \stackrel{\alpha}{\longrightarrow} \operatorname{Im} \phi_{S} .
$$

Thus $|G|=\operatorname{deg} \phi_{S} / \operatorname{deg} \alpha$. On the other hand, by [X1, Proposition 5.2], $\phi_{S}$ factors through $S \rightarrow P\left(f_{*} \omega_{S / C}\right)$ and $\operatorname{deg} \phi_{S} \leq 4$. So we have that $\operatorname{deg} \phi_{S}=4,\langle\sigma\rangle:=G$ is of order 2, and $\operatorname{Im} \phi_{S}$ is birationally isomorphic to $S /\langle\tau, \sigma\rangle$, where $\tau$ is the hyperelliptic involution of $f$. Clearly, $f$ is preserved under the action of $\sigma$. So there exists an isomorphism $\bar{\sigma} \in$ Aut $C$ such that $\bar{\sigma} \circ f=f \circ \sigma$. Since $g(C) \geq p_{g}(S) / 2-1 \geq 2$ and $\operatorname{Im} \phi_{S}$ is either a rational surface or a ruled surface over an elliptic curve by X1, Proposition 5.2], we have $\bar{\sigma}$ is not trivial. This implies that $\sigma$ acts non-trivially on $H^{0}\left(S, \Omega_{S}^{1}\right)$, and hence on $H^{1}(S, \mathbb{C})$, contradicting the assumption.

Theorem 3.2. Let $S$ be a complex non-singular projective surface of general type with $p_{g}(S) \geq 3$, and let $f: S \rightarrow C$ be a relatively minimal fibration of genus 2 . Assume that the canonical map $\phi_{S}$ of $S$ is composite with a pencil. Let $G \subset \operatorname{Aut} S$ be a subgroup of automorphisms of $S$, inducing trivial actions on $H^{2}(S, \mathbb{Q})$. Assume 
that $G$ is not trivial. Then

(i) $|G|=2$, and the generator $\sigma$ of $G$ is a bi-elliptic involution of $f$, i.e., $f \circ \sigma=f$, and for a general fiber $F$ of $f, \sigma_{\mid F}$ is a bi-elliptic involution of $F$.

(ii) Either $K_{S}^{2}=4 \chi\left(\mathcal{O}_{S}\right)$ and $q(S)=g(C)=1$, or $K_{S}^{2}=4 \chi\left(\mathcal{O}_{S}\right)-4, q(S)=1$ and $g(C)=0$.

Proof. By [X1, Theorem 5.1], $\phi_{S}$ factors through $f$ :

$$
\phi_{S}=\varphi \circ f: S \stackrel{f}{\longrightarrow} C \stackrel{\varphi}{\longrightarrow} \operatorname{Im} \phi_{S} \subset \mathbb{P}^{p_{g}(S)-1} .
$$

So $g(C) \leq 1$ by $\underline{\mathrm{X} 2}$. Let $d$ and $L$ be the degree and the hyperplane section of $\operatorname{Im} \phi_{S}$ in $\mathbb{P}^{p_{g}(S)-1}$, respectively. We have $d \geq \operatorname{codim} \operatorname{Im} \phi_{S}+1=p_{g}(S)-1$ (cf. [Mu $)$. Since $f^{*} \varphi^{*} L$ is the moving part of $\left|K_{S}\right|$,

$$
\begin{aligned}
p_{g}(S)=h^{0}\left(S, \varphi^{*} L\right) & =\operatorname{deg} \varphi d+1-g(C)+h^{1}\left(C, \varphi^{*} L\right) \\
& \geq \operatorname{deg} \varphi\left(p_{g}(S)-1\right) .
\end{aligned}
$$

So

$$
\begin{aligned}
& \operatorname{deg} \varphi=1, \\
& d= \begin{cases}\chi\left(\mathcal{O}_{S}\right) & \text { if } g(C)=1, \\
\chi\left(\mathcal{O}_{S}\right)+q(S)-2 & \text { if } g(C)=0 .\end{cases}
\end{aligned}
$$

By Hodge theory, $G$ acts trivially on $H^{0}\left(S, \omega_{S}\right)$. This implies that $G$ acts trivially on $\operatorname{Im} \phi_{S}$ and there is a homomorphism $h$ of $G$ into AutC. By (3.2.1), we have that Kerh $=G$, i.e. $G$ induces the trivial action on $C$, and $G \hookrightarrow \operatorname{Aut} F$ for a general fiber $F$ of $f$. Since $p_{g}(S / G)=p_{g}(S)>0$, the general fiber $F / G$ of $S / G \rightarrow C$ is not rational. Using the Hurwitz formula for $F \rightarrow F / G$, we get $|G|=2$.

The proof of (ii) of Theorem 3.2 is longer and is postponed until the next section.

In the rest of this section, we give examples to show that the two cases in (ii) of Theorem 3.2 really do occur.

Example 3.3 (Surfaces with $K_{S}^{2}=4 \chi\left(\mathcal{O}_{S}\right)$ ). Let $C^{\prime}$ and $E$ be elliptic curves, and let $\epsilon \in C^{\prime}$ be a non-trivial 2-torsion point. Take $X=\left(C^{\prime} \times E\right) / \mathbb{Z}_{2}$, where $\mathbb{Z}_{2}$ acts on $C^{\prime} \times E$ by $(c, e) \rightarrow(c+\epsilon,-e)$. Let

$$
p: X \rightarrow C:=C^{\prime} /\{0, \epsilon\} \text { and } q: X \rightarrow \mathbb{P}^{1}:=E /\{ \pm 1\}
$$

be fibrations induced by the projections of $C^{\prime} \times E$ to its two factors. Then $p$ is an elliptic fiber bundle and $q$ is an elliptic fibration with four double fibers $q^{*}\left(t_{i}\right)=2 C_{i}$ $(i=1, \cdots, 4)$, where $t_{i} \in \mathbb{P}^{1}$ are the image of 2-torsion points of $E$. Clearly, $C_{i}$ are sections of $p$. We have $K_{X}=p^{*} \eta$, where $\eta \in \operatorname{Pic}^{0} C$, which determines the double cover $C^{\prime} \rightarrow C$. Let $n \geq 2$ be an integer, and let $D$ be a divisor on $C$ of degree $n$. Let $\theta=p^{*} D+C_{1}$. Note that $2 \theta$ is the pullback of a very ample divisor on $C \times \mathbb{P}^{1}$ under the finite morphism $(p, q): X \rightarrow C \times \mathbb{P}^{1}$, and the linear system $|2 \theta|$ contains a smooth divisor $\Delta$. By Riemann-Hurwitz, $p_{\mid \Delta}: \Delta \rightarrow C$ has $8 n$ ramification points $q_{j}(j=1, \cdots, 8 n)$. Let $a_{j}=p\left(q_{j}\right)$. Since $C_{1}$ is a section of $p$, the set-theoretic intersection $\Delta \cap C_{1}$ is contained in the set of ramification points of $p_{\mid \Delta}$. After 
suitable re-indexing, we may assume that $\left\{q_{1}, \cdots, q_{2 n}\right\}=\Delta \cap C_{1}$ as a set. Let

$$
B=\Delta+\sum_{j=1}^{2 n} p^{*} a_{j}, \delta=p^{*}(2 D)+C_{1}
$$

Let $\pi^{\prime}: S^{\prime} \rightarrow X$ be the morphism associated with the singular double cover data $(B, \delta)$. $S^{\prime}$ has only canonical singularities. Let $\epsilon: S \rightarrow S^{\prime}$ be the minimal desingularization. We have

$$
K_{S}=\left(\pi^{\prime} \circ \epsilon\right)^{*}\left(p^{*}(\eta+2 D)+C_{1}\right)
$$

So $S$ has the following numerical invariants:

$$
K_{S}^{2}=8 n, p_{g}(S)=2 n, q(S)=1
$$

Now $f:=p \circ \pi^{\prime} \circ \rho: S \rightarrow C$ is a fibration of genus 2. Denote by $\tau$ the hyperelliptic involution of $f$, and by $\varsigma$ the involution of $S$ corresponding to $\pi^{\prime}$. Then $\langle\varsigma, \tau\rangle \cong$ $\mathbb{Z}_{2} \oplus \mathbb{Z}_{2}$. Take $\sigma=\tau \circ \varsigma$.

Claim. The involution $\sigma$ acts trivially on $\mathrm{H}^{i}(S, \mathbb{Q})$ for all $i$.

Proof of the Claim. Since $q(S)=g(C)$, by Hodge theory, $\sigma$ acts trivially on $H^{1}(S, \mathbb{Q})$. To check that the involution $\sigma$ acts trivially on $\mathrm{H}^{2}(S, \mathbb{Q})$, we analyse the action of $\sigma$ around the singular fibers of $f$.

Let $\rho: X^{\prime} \rightarrow X$ be the blow-up of $q_{j}(j=1, \cdots, 8 n)$. The exceptional curve $E_{1 j}^{\prime}=\rho^{*}\left(q_{j}\right)$ and the strict transform $\Delta^{\prime}$ of $\Delta$ meet transversely at point $q_{j}^{\prime}$. Let $\mu: X^{\prime \prime} \rightarrow X^{\prime}$ be the blow-up of $q_{j}^{\prime}(j=1, \cdots, 8 n)$. Let $E_{2 j}^{\prime \prime}=\mu^{*}\left(q_{j}^{\prime}\right)$ $(j=1, \cdots, 8 n)$ be the exceptional curves. We denote by $E_{1 j}^{\prime \prime}, p^{*}\left(a_{j}\right)^{\prime \prime}$ and $C_{1}^{\prime \prime}$ the strict transform of $E_{1 j}^{\prime}, p^{*}\left(a_{j}\right)$ and $C_{1}$ in $X^{\prime \prime}$, respectively. Set

$$
\begin{gathered}
B^{\prime \prime}=\mu^{*}\left(\rho^{*} B-2 \sum_{j=1}^{2 n} E_{1 j}^{\prime}\right)-2 \sum_{j=1}^{8 n} E_{2 j}^{\prime \prime}=\Delta^{\prime \prime}+\sum_{j=1}^{2 n} p^{*}\left(a_{j}\right)^{\prime \prime}+\sum_{j=2 n+1}^{8 n} E_{1 j}^{\prime \prime}, \\
\delta^{\prime \prime}=\mu^{*}\left(\rho^{*} \delta-\sum_{j=1}^{2 n} E_{1 j}^{\prime}\right)-\sum_{j=1}^{8 n} E_{2 j}^{\prime \prime}=(\rho \circ \mu)^{*}(2 D)+C_{1}^{\prime \prime}-\sum_{j=1}^{8 n} E_{2 j}^{\prime \prime} .
\end{gathered}
$$

We have that $B^{\prime \prime}$ is a smooth divisor on $X^{\prime \prime}$, and $B^{\prime \prime} \equiv 2 \delta^{\prime \prime}$. Let $\pi: S^{\prime \prime} \rightarrow X^{\prime \prime}$ be the morphism associated with the double cover data $\left(B^{\prime \prime}, \delta^{\prime \prime}\right)$. By the canonical resolution (cf. $\underline{\mathrm{Per}}$ ), we have a commutative diagram

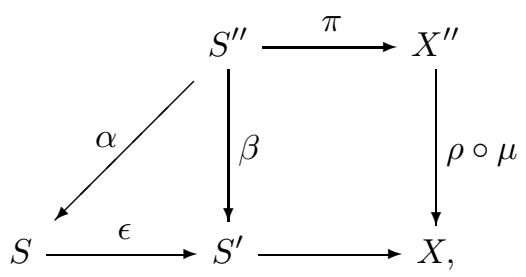

where $\beta$ is a desingularization of $S^{\prime}$, and $\alpha$ is the contraction of -1-curves on $S^{\prime \prime}$. Locally around a singular fiber,

$$
\pi:(f \circ \beta)^{*} a_{j} \rightarrow(p \circ \rho \circ \mu)^{*} a_{j}
$$


looks like this:

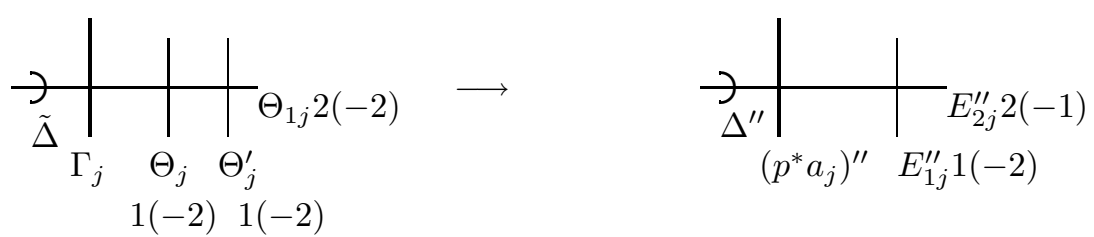

$1 \leq j \leq 2 n$
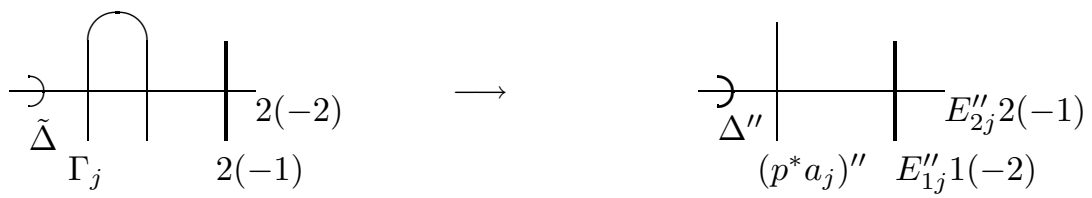

$$
2 n+1 \leq j \leq 8 n,
$$

where $\tilde{\Delta}$ is the inverse image of $\Delta^{\prime \prime}$, thick lines mean ramification or branch locus of $\pi$, and numbers without brackets are multiplicities and numbers within brackets denote self-intersections. Note that for $j=2 n+1, \cdots, 8 n,\left.\delta^{\prime \prime}\right|_{p^{*}\left(a_{j}\right)^{\prime \prime}}=\left.C_{1}^{\prime \prime}\right|_{p^{*}\left(a_{j}\right)^{\prime \prime}}-$ $\left.E_{2 j}^{\prime \prime}\right|_{p^{*}\left(a_{j}\right)^{\prime \prime}}$ is non-trivial, the inverse image $\Gamma_{j}$ of $p^{*}\left(a_{j}\right)^{\prime \prime}$ is connected. By the construction, $f$ has only $8 n$ singular fibers $f^{*} a_{j}(j=1, \cdots, 8 n)$, which have the following configurations:

- For $j=1, \cdots, 2 n, f^{*} a_{j}=\Theta_{j}+\Theta_{j}^{\prime}+2 \Theta_{1 j}+2 \Gamma_{j}$ is as in $\left(b_{1}\right)$ of $2.6 . \Gamma_{j}$ is an $\varsigma$-fixed elliptic curve.

- For $j=2 n+1, \cdots, 8 n, f^{*} a_{j}$ is an irreducible curve with exactly one node $p_{j}$, which is a non-isolated $\varsigma$-fixed point. The normalization of $f^{*} a_{j}$ is an elliptic curve.

Since $p_{j}$ is a non-isolated $\tau$-fixed point by [Ca1, Lemma 2.4] for $j=2 n+1, \cdots, 8 n$, both $\tau$ and $\varsigma$ exchange the local branches at $p_{j}$. So $\sigma$ fixes the local branches at $p_{j}$. This implies $p_{j}$ is an isolated fixed point of $\sigma$ for $j=2 n+1, \cdots, 8 n$.

For for $j=1, \cdots, 2 n$, since $\varsigma_{\mid \Gamma_{j}}$ is trivial, $\varsigma_{\mid \Theta_{1 j}}$ is an involution with fixed points $t_{j}=\Gamma_{j} \cap \Theta_{1 j}, s_{j}=\tilde{\Delta} \cap \Theta_{1 j}$ (see the picture above). Since $\tilde{\Delta}$ is $\tau$-invariant, $s_{j}$ is $\tau$-fixed. From

$$
\alpha^{*} K_{S}=\pi^{*}(\rho \circ \mu)^{*}\left(p^{*}(\eta+2 D)+C_{1}\right),
$$

we easily see that $\Theta_{j}+\Theta_{j}^{\prime}+\Theta_{1 j}$ is contained in the fixed part of $\left|K_{S}\right|$. By 2.9, $f^{*} a_{j}$ is not of type $\mathrm{V}$ in the sense of Horikawa. So by $2.8, t_{j}, s_{j}$ are isolated $\tau$-fixed points and there are three non-isolated $\tau$-fixed points $r_{1 j}, r_{2 j}, r_{3 j}$ on $\Gamma_{j}$. So $\Theta_{j}$ is $\sigma$-fixed (otherwise, $\langle\varsigma, \tau\rangle \hookrightarrow$ Aut $\Theta_{1 j}$, a contradiction by 2.2) and $r_{1 j}, r_{2 j}, r_{3 j}$ are $\sigma$-fixed points.

Let $H$ be the $\sigma$-fixed divisor which is horizontal with respect to $f$. We show that $f_{\mid H}: H \rightarrow C$ is étale. In particular, this implies that $r_{1 j}, r_{2 j}, r_{3 j}$ are isolated $\sigma$-fixed points. Suppose $p \in H$ is a ramification point of $f_{\mid H}$. Let $F^{\prime}=f^{*}(f(p))$. Since $H F^{\prime}=2$, we have $H \cap F^{\prime}=\{p\}$. Since $H$ is $\varsigma$-invariant, we have $p$ is $\langle\tau, \varsigma\rangle$-fixed. Since $\langle\tau, \varsigma\rangle$ is not cyclic, $p$ is a singular point of $F^{\prime}$ by 2.2. If $F^{\prime}=f^{*}\left(a_{j}\right)$ for some $j, 2 n+1 \leq j \leq 8 n$, then $p=p_{j}$, which is a contradiction since $p_{j}$ is an isolated fixed point of $\sigma$. Now we suppose $F^{\prime}=f^{*}\left(a_{j}\right)$ for some $j, 1 \leq j \leq 2 n$. Since $\Theta_{1 j}$ is $\sigma$-fixed, $\Theta_{j}$ is not $\sigma$-fixed. So there is a $\sigma$-fixed point $o_{j}$ on $\Theta_{i j} \backslash \Theta_{j} \cap \Theta_{1 j}$. By 
Ca1 Lemma 2.4], $H$ passes through $o_{j}$, which is a contradiction. Summing up, we have

$$
\begin{aligned}
S^{\sigma}=\left\{p_{j} ; j=2 n+1, \cdots, 8 n\right\} & \cup\left\{r_{1 j}, r_{2 j}, r_{3 j} ; j=1, \cdots, 2 n\right\} \\
& \cup\left\{\Theta_{1 j} ; j=1, \cdots, 2 n\right\} \cup\{H\} .
\end{aligned}
$$

Since $H$ is étale over $C, e(H)=0$. So

$$
e\left(S^{\sigma}\right)=6 n+3 \times 2 n+2 \times 2 n=16 n .
$$

By the Noether formula, $e(S)=16 n$. So by the topological Lefschetz formula (cf. [AS, p. 566]), we get $\operatorname{dim} H^{2}(S, \mathbb{Q})^{\sigma}=\operatorname{dim} H^{2}(S, \mathbb{Q})$, i.e. $\sigma$ acts trivially on $H^{2}(S, \mathbb{Q})$.

Example 3.4 (Surfaces with $K_{S}^{2}=4 \chi\left(\mathcal{O}_{S}\right)-4$ ). Let $E$ be an elliptic curve, and let $e \in E$ be a point. Let $X=\mathbb{P}^{1} \times E$, and $p: X \rightarrow \mathbb{P}^{1}, q: X \rightarrow E$ the the projections. Let $n \geq 2$ be an integer, and let $D$ be a divisor on $\mathbb{P}^{1}$ of degree $n$. Let $\theta=p^{*} D+C$, where $C=\mathbb{P}^{1} \times e$ is a section of $p$. Let $\Delta \in|2 \theta|$ be a smooth divisor. By Riemann-Hurwitz, $p_{\mid \Delta}: \Delta \rightarrow \mathbb{P}^{1}$ has $8 n$ ramification points $q_{j}(j=1, \cdots, 8 n)$. Let $a_{j}=p\left(q_{j}\right)$. Since $C$ is a section of $p$, the set-theoretic intersection $\Delta \cap C$ is contained in the set of ramification points of $p_{\mid \Delta}$. After suitable re-indexing, we may assume that $\left\{q_{1}, \cdots, q_{2 n}\right\}=\Delta \cap C$ as a set. Let

$$
B=\Delta+\sum_{j=1}^{2 n} p^{*} a_{j}, \delta=p^{*}(2 D)+C .
$$

Let $\pi^{\prime}: S^{\prime} \rightarrow X$ be the morphism associated with the singular double cover data $(B, \delta)$. $S^{\prime}$ has only canonical singularities. Let $\epsilon: S \rightarrow S^{\prime}$ be the minimal desingularization. We have $K_{S}=\left(\pi^{\prime} \circ \epsilon\right)^{*}\left(p^{*}\left(K_{\mathbb{P}^{1}}+2 D\right)+C\right)$. So $S$ has the following numerical invariants:

$$
K_{S}^{2}=8 n-8, p_{g}(S)=2 n-1, q(S)=1 .
$$

Now $f:=p \circ \pi^{\prime} \circ \rho: S \rightarrow \mathbb{P}^{1}$ is a fibration of genus 2. Denote by $\tau$ the hyperelliptic involution of $f$, and by $\varsigma$ the involution of $S$ corresponding to $\pi^{\prime}$. Take $\sigma=\tau \circ \varsigma$. Clearly $\sigma$ induces -id on $\mathrm{H}^{1}(S, \mathbb{Q})$. We can verify as in 3.3 that the involution $\sigma$ acts trivially on $H^{2}(S, \mathbb{Q})$.

\section{Proof of Theorem 3.2(ii)}

Let me start by fixing notations.

4.1. Let $f: S \rightarrow C$ be as in Theorem 3.2, and let $\tau$ be the hyperelliptic involution of $f$. Let $\sigma$ be as in Theorem [3.2(i), let $\lambda=\sigma \circ \tau$, and let $G^{\prime} \subset$ Aut $S$ be the subgroup generated by $\sigma$ and $\tau$. We have that $\lambda$ is a bi-elliptic involution of $f$, and $G^{\prime} \simeq \mathbb{Z}_{2} \oplus \mathbb{Z}_{2}$.

Let $f_{\lambda}: S_{\lambda} \rightarrow C$ be the relatively minimal model of a desingularization of $S / \lambda \rightarrow$ $C$. Then $f_{\lambda}: S_{\lambda} \rightarrow C$ is an elliptic fibration. We employ the standard notation of Kodaira of singular elliptic fibres.

We write $\left|K_{S}\right|=|M|+H+V$, where $M=f^{*} L$ for some $L \in \operatorname{Pic} C$ is the moving part of $\left|K_{S}\right|$, and $H$ (resp. $V$ ) is the horizontal part (resp. the vertical part) of the fixed part of $\left|K_{S}\right|$.

For any two curves $C$ and $D$ on $S$, we denote by $C \cap D$ the set-theoretic intersection $\operatorname{supp} C \cap \operatorname{supp} D$. 
Lemma 4.2. Let $F^{\prime}$ be a singular fiber of $f$ and $D<F^{\prime}$ is an irreducible curve. Assume that one of the following is true: (1) $F^{\prime}$ is 2-connected; (2) $D<\Gamma_{i}$ for $i=1$ or 2 if $F^{\prime}$ is as in $\left(a_{k}\right)$ of 2.6; (3) $D<\Gamma$ if $F^{\prime}$ is as in $\left(b_{k}\right)$ of 2.6. Then $D$ is $\sigma$-invariant but not $\sigma$-fixed.

Proof. By 2.9, $V=\sum_{\text {singular fiber } F^{\prime}} \Delta_{F^{\prime}}$, where $V$ is as above and $\Delta_{F^{\prime}}$ is as in 2.9. The result follows by 2.3 and 2.9 .

Lemma 4.3. Notation as in 4.1. We have $H$ is reduced, $\sigma$-fixed, and $\tau$-invariant but not $\tau$-fixed.

Proof. Let $F$ be a general fiber of $f$. Suppose $H=2 D$ for some irreducible curve $D \subset S$. Then $D F=1$. Let $p=D \cap F$. Since $H$ is $\sigma$-invariant, $p$ is $\sigma$-fixed. From $K_{F}=K_{S \mid F} \equiv H_{\mid F}=2 p$, we have that $p$ is $\tau$-fixed. So $p \in F$ is $G^{\prime}$-fixed. This is a contradiction since $G^{\prime}$ is not cyclic. Hence $H$ is reduced.

Since $\sigma$ has two non-isolated fixed points on $F$, by 2.3. we have that $H$ is $\sigma$-fixed. Note that $\tau(H)$ is also $\sigma$-fixed and there are exactly two $\sigma$-fixed points on $F$; we have $\tau(H)=H$. By 2.2, $H$ is not $\tau$-fixed.

Lemma 4.4. Let $f: S \rightarrow C$ and $\sigma$ be as in 4.1. Let $F^{\prime}$ be a singular fiber of $f$, and let $\Theta<F^{\prime}$ be a connected-2-cycle. Assume that one of the following is true: (1) $F^{\prime}$ is 2-connected; (2) there is an elliptic tail $\Gamma<F^{\prime}$ such that $\Theta<\Gamma$. Then $\Theta$ is of type $A_{n}$.

Proof. Suppose that $\Theta$ is of type $D_{n}$ or $E_{n}$. Let $\Theta^{\prime}<\Theta$ be the irreducible component such that $\Theta^{\prime}$ meets $\operatorname{supp}(\Theta)-\Theta^{\prime}$ in three distinct points. By Ca2, Lemma 3.1], there exist three $\sigma$-fixed points on $\Theta^{\prime}$. This implies that $\Theta^{\prime}$ is $\sigma$-fixed. By 2.3, $\Theta^{\prime}<\Delta_{F^{\prime}}$. This is a contradiction by 2.9.

Lemma 4.5. Let $f: S \rightarrow C, H$ and $\sigma$ be as in 4.1, and let $F^{\prime}$ be a singular fiber of $f$. Assume that $F^{\prime}$ is 2-connected. Then

(i) For any-2-curve $\Theta<F^{\prime}$, we have $\Theta \cap H=\emptyset$.

(ii) For any irreducible curve $\Gamma<F^{\prime}, \sigma_{\mid \Gamma}$ is not trivial. In particular, $\sigma$ has exactly two (resp. four) fixed points on the normalization $\tilde{\Gamma}$ provided $g(\tilde{\Gamma})=0(\operatorname{resp} . g(\tilde{\Gamma})=1)$.

(iii) If $q \in F^{\prime}$ is $\sigma$-fixed, then either $q \in H$ or $q$ is isolated $\sigma$-fixed.

Proof. (i) By 2.9, $K_{S}=M+H+\sum_{\text {singular fiber } F^{\prime \prime}} \Delta_{F^{\prime \prime}}$, and $\Delta_{F^{\prime \prime}}=0$ if $F^{\prime \prime}$ is 2-connected. So $H \Theta=K_{S} \Theta-\Delta_{F^{\prime}} \Theta=0$ for any -2-curve $\Theta<F^{\prime}$.

(ii) and (iii) follows by 4.2 .

Lemma 4.6. Let $f: S \rightarrow C$ and $\sigma$ be as in 4.1. Then $f$ has no singular fibers of type $\left(a_{k}\right)$ of 2.6 .

Proof. Suppose that $f$ has a singular fiber $F^{\prime}=\Gamma_{1}+\Theta_{1}+\cdots+\Theta_{k}+\Gamma_{2}$ of type $\left(a_{k}\right)$. Let $p=\Gamma_{1} \cap \Theta_{1}$ if $k>0$, otherwise $p=\Gamma_{1} \cap \Gamma_{2}$. By 2.8, $p$ is an isolated $\tau$-fixed point. Let $\Gamma<\Gamma_{1}$ be the irreducible curve passing through $p$. Then $\Gamma$ is $\tau$-invariant, and $p \in \Gamma$ is a smooth point of $\Gamma$. By Lemma 4.2, $\Gamma$ is $\sigma$-invariant but not $\sigma$-fixed, and $p$ is $\sigma$-fixed. By 2.4 $G \hookrightarrow \operatorname{Aut} \Gamma$, which is a contradiction by 2.2 , 
Lemma 4.7. Let $f: S \rightarrow C$ and $\sigma$ be as in 4.1. Let $F^{\prime}$ be a singular fiber of $f$. Assume that $F^{\prime}=\Theta+\Theta^{\prime}+2 \Theta_{1}+\cdots+2 \Theta_{k}+2 \Gamma$ is of type $\left(b_{k}\right)$ of 2.6 , Then

(i) $f_{\mid H}: H \rightarrow C$ is étale at $F^{\prime} \cap H$.

(ii) $k$ is odd, $F^{\prime}$ is not of type $V$ in the sense of Horikawa, and $\Gamma$ is either a smooth elliptic curve or two smooth rational curves meeting transversally in two points.

(iii) $\Theta_{1}, \Theta_{3}, \cdots, \Theta_{k-2}, \Theta_{k}$ are $\sigma$-fixed curves, and there are exactly three isolated $\sigma$-fixed points on $\Gamma$.

Proof. (i) First we exclude the case $k=0$. If $k=0$, let $\Gamma^{\prime}<\Gamma$ be the -3 -curve if $\Gamma$ is reducible, otherwise $\Gamma^{\prime}=\Gamma$ (cf. 2.5). Then $\Gamma^{\prime}$ intersects both $\Theta$ and $\Theta^{\prime}$. Set $t=\Theta \cap \Gamma^{\prime}$ and $t^{\prime}=\Theta^{\prime} \cap \Gamma^{\prime}$. By Lemma 4.2, $\sigma \neq$ id on $\Gamma^{\prime}$. By [Ca2, Lemma 3.1], $t$ and $t^{\prime}$ are $\sigma$-fixed points. By 2.7 and 2.8 $\tau(\Theta)=\Theta^{\prime}$, and there is precisely an isolated $\tau$-fixed point $p$ on $\Gamma^{\prime}$. Then $p$ is a $\sigma$-fixed point. Summing up, we have $G^{\prime} \hookrightarrow$ Aut $\Gamma^{\prime}$ and $p$ is $G^{\prime}$-fixed. By 2.2. we can assume $p \in \Gamma^{\prime}$ is a singular point of $\Gamma^{\prime}$. Then $p$ is an isolated $\sigma$-fixed point since $H \Gamma^{\prime} \leq 1$. This implies there are at least three $\sigma$-fixed points on the normalization of $\Gamma^{\prime}$. So $\sigma=$ id on $\Gamma^{\prime}$, a contradiction.

Now we can assume $k>0$. By [Ca2, Lemma 3.1], it is easy to see $\Theta_{1}$ is $\sigma$-fixed. Thus $\sigma_{\mid \Theta}$ is non-trivial, and hence there is a $\sigma$-fixed point $q$ on $\Theta \backslash \Theta \cap \Theta_{1}$. By [Ca1, Lemma 2.4], $q$ is a non-isolated $\sigma$-fixed point. Similarly, there is a non-isolated $\sigma$ fixed point $q^{\prime}$ on $\Theta^{\prime} \backslash \Theta^{\prime} \cap \Theta_{1}$. By 2.3, we have $H$ passes through $q$ and $q^{\prime}$, i.e., $f_{\mid H}$ is étale at $H \cap F^{\prime}$.

(ii) By the proof of (i), we have $k>0$ and $\Theta_{1}$ is $\sigma$-fixed. By 2.3 and 2.9, $F^{\prime}$ is not of type $\mathrm{V}$ in the sense of Horikawa.

Now we show that $k$ is odd. Otherwise, set $k=2 n$. Let $t_{i}=\Theta_{i} \cap \Theta_{i+1}$ $(i=1, \cdots, k-1)$ and $t_{k}=\Theta_{k} \cap \Gamma$. By [Ca2, Lemma 3.1] and 2.8, $t_{i}(i=1, \cdots, k)$ are $\sigma$-fixed and isolated $\tau$-fixed points. Clearly for $i=1, \cdots, k, \Theta_{i}$ is $G^{\prime}$-invariant and the kernel of $G^{\prime} \rightarrow$ Aut $\Theta_{i}$ is not trivial by 2.2. So we have $\Theta_{1}, \Theta_{3}, \cdots, \Theta_{2 n-1}$ are $\sigma$-fixed. By Lemmas 4.2 and 4.7 (i), $t_{2 n}$ is an isolated $\sigma$-fixed point.

Let $\rho: \tilde{S} \rightarrow S$ be the blowup of all isolated fixed points of $\sigma$, and let $\tilde{\sigma}$ be the induced involution on $\tilde{S}$. Let $\tilde{\Theta}_{2 n}$ be the strict transform of $\Theta_{2 n}$ in $\tilde{S}$, and let $D$ be the image of $\tilde{\Theta}_{2 n}$ under the quotient map $\pi: \tilde{S} \rightarrow \tilde{S} / \tilde{\sigma}$. Since there is precisely one isolated $\sigma$-fixed point on $\Theta_{2 n}, \tilde{\Theta}_{2 n}^{2}=-3$. On the other hand, from $\tilde{\Theta}_{2 n}=\pi^{*} D$, we get $\tilde{\Theta}_{2 n}^{2}=2 D^{2}$. This is a contradiction. So $k$ is odd.

Now we show that $\Gamma$ is one of the types described in the lemma. By 2.5, we distinguish between four cases:

1) $\Gamma$ is a rational curve with a single node $p$. By Lemma 4.7(i), $p$ is an isolated $\sigma$-fixed point. So there exist three $\sigma$-fixed points (the inverse image of $p$ and $\Theta_{k} \cap \Gamma$ ) on the normalization $\tilde{\Gamma}$ of $\Gamma$. This implies $\sigma$ acts trivially on $\tilde{\Gamma}$, and hence on $\Gamma$. This is a contradiction by Lemma 4.2 .

2) $\Gamma$ is a rational curve with a single cusp $p$. In this case $p$ is $G^{\prime}$-fixed and $G^{\prime} \hookrightarrow$ Aut $\Gamma$. Since $G^{\prime}$ is not cyclic, this is impossible by 2.2

3) $\Gamma=\Gamma_{1}+\Theta$ is of type (ii) of 2.5 . Then $\Theta$ a -2-cycle of type $A_{n}$ with $n \geq 1$. If $n \geq 2$, we get a contradiction as in 1$)$.

4) $\Gamma=\Gamma_{1}+\Theta$ is of type (iii) of 2.5. By Lemma 4.4, we can assume $\Theta$ is a -2-curve and $\Gamma_{1}$ and $\Theta$ meet in one point $p$. We claim $\Theta$ is not $\tau$-fixed. Otherwise, let $R$ be the horizontal part of the $\tau$-fixed curves. Then $R$ intersects $\Gamma_{1}$ at points other than $p$ and $\Theta_{k} \cap \Gamma$. This implies $\Gamma_{1}$ and hence $\Gamma_{1}+R$ are $\tau$-fixed, which is a contradiction by 2.1. By Lemma 4.2 $\sigma_{\mid \Theta} \neq$ id. Since $p$ is $G^{\prime}$-fixed and $G^{\prime}$ is not 
cyclic, by [2.2, $\lambda_{\mid \Theta}=$ id. Similarly we have $\lambda_{\mid \Gamma_{1}}=$ id. So $\lambda_{\mid \Gamma_{1}+\Theta}=$ id, which is a contradiction by 2.1 .

(iii) By the proof of (ii), $\Theta_{1}, \Theta_{3}, \cdots, \Theta_{k-2}, \Theta_{k}$ are $\sigma$-fixed curves. If $\Gamma$ is smooth, since $\sigma_{\mid \Gamma} \neq$ id by Lemma 4.2, $\sigma$ has four fixed points $r_{0}:=\Gamma \cap \Theta_{k}, r_{1}, r_{2}, r_{3}$ on $\Gamma$. By Lemma 4.7(i), $r_{i}(i=1,2,3)$ are isolated $\sigma$-fixed points. Now we may assume $\Gamma=\Gamma_{1}+\Theta$ are two smooth rational curves meeting transversally in two points $p_{i}$ $(i=1,2)$. We have $\sigma\left(p_{1}\right)=p_{2}$ (otherwise, we get a contradiction as in 1) above). Since $\sigma_{\mid \Gamma_{1}} \neq$ id and $\sigma_{\mid \Theta} \neq$ id, we have $\sigma$ has a single isolated fixed point on $\Gamma_{1}$, and exactly two isolated fixed points on $\Theta$.

Corollary 4.8. Let $f: S \rightarrow C, \sigma, \lambda$ and $f_{\lambda}: S_{\lambda} \rightarrow C$ be as in 4.1. Let $F^{\prime}$ be a singular fibre of $f$. Assume that $F^{\prime}$ is not 2-connected. Then $F^{\prime}=\Theta+\Theta^{\prime}+$ $2 \Theta_{1}+\cdots+2 \Theta_{k}+2 \Gamma$ ( $k$ is odd) is not of type $V$ in the sense of Horikawa, and $\Gamma$ is either a smooth elliptic curve or two smooth rational curves meeting transversally in two points. The configuration for $F_{\lambda}^{\prime}:=f_{\lambda}^{*} f\left(F^{\prime}\right)$ is of type $\mathrm{I}_{0}$ or $\mathrm{I}_{1}$ depending on whether $\Gamma$ is smooth or not.

Proof. By Lemmas 4.6 and 4.7, we have that $F^{\prime}$ is of the type described in the corollary. Now we show that, if $\Gamma$ is smooth, then $F_{\lambda}^{\prime}=f_{\lambda}^{*} f\left(F^{\prime}\right)$ is of type $\mathrm{I}_{0}$. The proof of the other case is similar. By the proof of Lemma 4.7 (iii), $\Theta_{2}, \Theta_{4}, \cdots, \Theta_{k-3}, \Theta_{k-1}$ and $\Gamma$ are $\lambda$-fixed, $\lambda(\Theta)=\Theta^{\prime}$, and there are no isolated $\lambda$-fixed points on $F^{\prime}$. Let $h: \tilde{S} / \tilde{\lambda} \rightarrow C$ be as in 2.4. We have that $h^{*}\left(f\left(F^{\prime}\right)\right)$ has the following configuration (numbers without brackets are multiplicities, and numbers within brackets denote self-intersections):

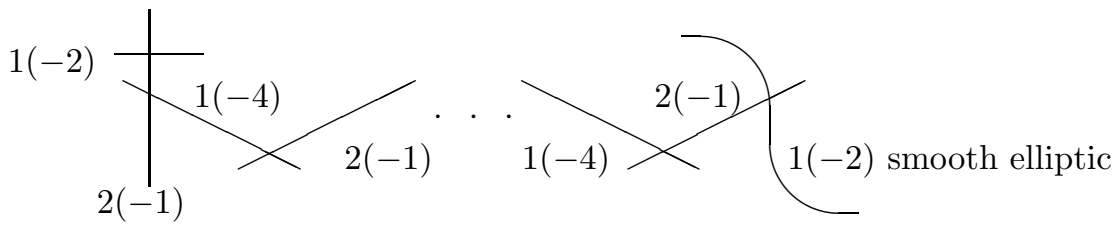

Blowing down -1-curves contained in $h^{*}\left(f\left(F^{\prime}\right)\right)$, we have that $f_{\lambda}^{*}\left(f\left(F^{\prime}\right)\right)$ is a smooth elliptic curve.

Lemma 4.9. Let $f: S \rightarrow C, H$ and $\sigma$ be as in 4.1, and let $F^{\prime}$ be a singular fiber of $f$. Assume that $F^{\prime}$ is 2-connected and $f_{\mid H}: H \rightarrow C$ is étale at $H \cap F^{\prime}$. Then the possible configurations for $F^{\prime}$ and the action of $\sigma$ on $F^{\prime}$ are as follows:

(i) $F^{\prime}$ is an irreducible curve with a single node $p$, which is an isolated $\sigma$-fixed point;

(ii) $F^{\prime}$ is an irreducible curve with exactly two nodes $p_{i}(i=1,2) . \sigma\left(p_{1}\right)=p_{2}$;

(iii) $F^{\prime}$ is an irreducible curve with exactly two cusps $p_{i}(i=1,2) . \sigma\left(p_{1}\right)=p_{2}$;

(iv) $F^{\prime}$ is an irreducible curve with a tacnode $p$, which is an isolated $\sigma$-fixed point;

(v) $F^{\prime}=\Gamma+\Theta$, where $\Gamma$ is an smooth elliptic curve and $\Theta$ is a-2-cycle of type $A_{n}(n \geq 1)$. $\Gamma$ and $\Theta$ meet transversally in two points $q_{j}(j=1,2)$, which are isolated $\sigma$-fixed points;

(vi) $F^{\prime}=\Gamma_{1}+\Gamma_{2}+\Theta$, where $\Gamma_{i}$ are -3 -curves meeting transversally in two points $q_{i}, \sigma\left(q_{1}\right)=q_{2}$, and $\Theta$ is a-2-cycle of type $A_{n}$. For $i=1,2, \Gamma_{i}$ and $\Theta$ meet transversally in one point $s_{i}$, which is an isolated $\sigma$-fixed point; 
(vii) $F^{\prime}=\Gamma_{1}+\Gamma_{2}$, where $\Gamma_{i}$ are irreducible smooth curves meeting transversally in three points $p_{i}(i=1,2,3)$. Among them, one, say $p_{3}$, is an isolated $\sigma$-fixed point. $\sigma\left(p_{1}\right)=p_{2}$;

(viii) $F^{\prime}=\Gamma_{1}+\Gamma_{2}$, where $\Gamma_{i}$ are irreducible smooth curves meeting in one point $p$, which is an isolated $\sigma$-fixed point, and $\Gamma_{1} \Gamma_{2}=3$.

Proof. The assumption implies that $H \cap F^{\prime}$ consists of two smooth points of $F^{\prime}$, say $p_{1}$ and $p_{2}$. We distinguish between three cases:

1) $F^{\prime}$ is irreducible. Note that $G^{\prime} \hookrightarrow$ Aut $F^{\prime}$ by 2.1, and the normalization of $F^{\prime}$ has no $G^{\prime}$-fixed points by 2.2. We have $F^{\prime}$ as in (i)-(iv) of Lemma 4.9 ,

2) $F^{\prime}$ is reducible, and there is an irreducible component $\Gamma<F^{\prime}$ with $p_{a}(\Gamma)=1$. Since $F^{\prime}$ is 2 -connected, we have $K_{S} \Gamma=2, \Gamma^{2}=-2$. Thus $F^{\prime}-\Gamma$ consists of -2-curves. By Lemma 4.5(i), $p_{i} \in \Gamma$ for $i=1$ and 2. We claim that $\Gamma$ is smooth. Otherwise, suppose $q \in \Gamma$ is a singular point. Then $q$ is an isolated $\sigma$-fixed point by Lemma 4.5)(iii). So $\sigma$ has at least one fixed point on the normalization of $\Gamma$ except for $p_{1}$ and $p_{2}$. This implies $\sigma=$ id on $\Gamma$, and hence on $\Gamma+H$, a contradiction by 2.1. Since $\sigma_{\mid \Gamma}$ is not a translation of $\Gamma$, it has two fixed points $q_{j}(j=1,2)$ except $p_{1}$ and $p_{2} . q_{j}$ are isolated $\sigma$-fixed points by Lemma 4.5(iii). By [Ca1, Lemma 2.4], $q_{j} \in F^{\prime}$ is singular. So we have $\Gamma$ and $F^{\prime}-\Gamma$ meet transversally in two points $q_{j}$ $(j=1,2)$, i.e. $F^{\prime}$ is in (v) of Lemma 4.9.

3) $F^{\prime}$ is reducible, and any irreducible component $\Gamma<F^{\prime}$ is smooth rational. First we note that, if $\Gamma<F^{\prime}$ is an irreducible component with $K_{S} \Gamma>0$, then mult $_{\Gamma} F^{\prime}=1$. Otherwise, $F^{\prime}=2 \Gamma+$ some -2 -curves. By Lemma 4.5(i), $p_{1}, p_{2} \in \Gamma$, which is a contradiction since $p_{i}$ are smooth points of $F^{\prime}$. So we have either there is an irreducible component $\Gamma<F^{\prime}$ with $K_{S} \Gamma=2$, or there are two irreducible components $\Gamma_{i}<F^{\prime}(i=1,2)$ with $K_{S} \Gamma_{1}=K_{S} \Gamma_{2}=1$. In the former case, by Lemma 4.5(i), $p_{1}, p_{2} \in \Gamma$. By Lemmas 4.5(ii) and 4.4, we have $F^{\prime}=\Gamma+\Theta_{1}+\Theta_{2}$, where $\Theta_{i}$ are -2 -curves, and $\Gamma$ and $\Theta_{i}$ meet transversally in two points $q_{i 1}$ and $q_{i 2}$, and $\sigma\left(q_{i 1}\right)=q_{i 2}$. Then $\sigma$ has two isolated fixed points on $\Theta_{i}$, which is impossible by [Ca1, Lemma 2.4]. In the latter case, we have $0 \leq \Gamma_{1} \Gamma_{2} \leq 3$. If $\Gamma_{1} \Gamma_{2}=0$ or 1 , by Lemma 4.5(ii), the possible configurations for $F^{\prime}$ do not occur. If $\Gamma_{1} \Gamma_{2}=2$, by Lemma 4.5(ii), $\Gamma_{1}$ and $\Gamma_{2}$ meet transversally in two points $q_{i}$ and $\sigma\left(q_{1}\right)=q_{2}$. So $F^{\prime}$ is in (vi) of Lemma 4.9. If $\Gamma_{1} \Gamma_{2}=3$, by Lemma 4.5(ii), $F^{\prime}$ is in (vii)-(viii) of Lemma 4.9

Lemma 4.10. Let $f: S \rightarrow C, H$ and $\sigma$ be as in 4.1, and let $F^{\prime}$ be a singular fiber of $f$. Assume that $F^{\prime}$ is 2-connected and $f_{\mid H}: H \rightarrow C$ is not étale at $H \cap F^{\prime}$. Then the possible configurations for $F^{\prime}$ and the action of $\sigma$ on $F^{\prime}$ are as follows:

(i) $F^{\prime}$ is an irreducible curve with a single node $p$, which is a non-isolated $\sigma$-fixed point;

(ii) $F^{\prime}$ is an irreducible curve with exactly two nodes, one is an isolated $\sigma$-fixed point and the other is a non-isolated $\sigma$-fixed point;

(iii) $F^{\prime}$ is an irreducible curve with a tacnode $p$, which is a non-isolated $\sigma$-fixed point;

(iv) $F^{\prime}=\Gamma+\Theta$, where $\Gamma$ is an irreducible rational curve with a single node $p$, which is a non-isolated $\sigma$-fixed point, and $\Theta a-2$-cycle of type $A_{n} . \Gamma$ and $\Theta$ meet transversally in two points $p_{i}(i=1,2)$, which are isolated $\sigma$-fixed points; 
(v) $F^{\prime}=\Gamma_{1}+\Gamma_{2}$, where $\Gamma_{i}$ are irreducible smooth curves meeting in two points $p$ and $q . p \in F^{\prime}$ is a tacnode, which is a non-isolated $\sigma$-fixed point. $q$ is a node, which is an isolated $\sigma$-fixed point;

(vi) $F^{\prime}=\Gamma_{1}+\Gamma_{2}+\Theta$, where $\Gamma_{i}$ are irreducible smooth curves meeting in one point $p$, which is a non-isolated $\sigma$-fixed point, with $\Gamma_{1} \Gamma_{2}=2$, and $\Theta a$ -2-cycle of type $A_{n}$. For $i=1$ and $2, \Gamma_{i}$ and $\Theta$ meet transversally in one point $p_{i}$, which is an isolated $\sigma$-fixed point.

Proof. The proof is similar to that of Lemma 4.9, and left to the reader.

Corollary 4.11. Let $f: S \rightarrow C, H, \sigma, \lambda$ and $f_{\lambda}: S_{\lambda} \rightarrow C$ be as in 4.1, and let $F^{\prime}$ be a singular fiber of $f$. Assume that $F^{\prime}$ is 2-connected and $F_{\lambda}^{\prime}$ is the fiber of $f_{\lambda}$ over $f\left(F^{\prime}\right)$. Then

(i) If $f_{\mid H}: H \rightarrow C$ is étale at $H \cap F^{\prime}$, then the possible configurations for $F_{\lambda}^{\prime}$ are as follows:

\begin{tabular}{|l|l|l|l|l|l|l|l|l|}
\hline$F^{\prime}$ is as in () of 4.9 & i & ii & iii & iv & v & vi & vii & viii \\
\hline$F_{\lambda}^{\prime}$ & $\mathrm{I}_{0}$ & $\mathrm{I}_{1}$ & II & $\mathrm{I}_{1}$ or III & $\mathrm{I}_{0}$ & $\mathrm{I}_{1}$ & $\mathrm{I}_{1}$ & II \\
\hline
\end{tabular}

(ii) If $f_{\mid H}: H \rightarrow C$ is not étale at $H \cap F^{\prime}$, then the possible configurations for $F_{\lambda}^{\prime}$ are as follows:

\begin{tabular}{|l|l|l|l|l|l|l|}
\hline$F^{\prime}$ is as in () of 4.10 & $\mathrm{i}$ & ii & iii & iv & v & vi \\
\hline$F_{\lambda}^{\prime}$ & $\mathrm{I}_{2}$ & $\mathrm{I}_{2}$ & $\mathrm{I}_{1}$ or III & $\mathrm{I}_{2}$ & III & III \\
\hline
\end{tabular}

Proof. By Lemmas 4.9 and 4.10, the proof is similar to that of Corollary 4.8 and is left to the reader. (We note that, if $F^{\prime}$ is as in (iv) of Lemma 4.9 or (iii) of Lemma 4.10. $f_{\lambda}^{*}\left(f\left(F^{\prime}\right)\right)$ is $\mathrm{I}_{1}$ or III depending on whether $p \in F^{\prime}$ is non-isolated $\lambda$-fixed or isolated $\lambda$-fixed.)

Proposition 4.12. Let $f_{\lambda}: S_{\lambda} \rightarrow C$ be as in 4.1. Then $f_{\lambda}: S_{\lambda} \rightarrow C$ is an elliptic fiber bundle.

Proof. By Lemma 4.3, $H$ is $\lambda$-invariant but not $\lambda$-fixed. So $H / \lambda \hookrightarrow S / \lambda$ is a section of the elliptic fibration $f_{\lambda}$. So $f_{\lambda}$ has no multiple fibers. By the canonical divisor formula for the elliptic fibration (note that $p_{g}\left(S_{\lambda}\right)=0$ and $g(C) \leq 1$ ), we have $\kappa\left(S_{\lambda}\right) \neq 1$. By the Noether formula,

$$
12 \chi\left(\mathcal{O}_{S_{\lambda}}\right)=e\left(S_{\lambda}\right)=\sum_{\text {singular fiber } F^{\prime}} e\left(F^{\prime}\right) \geq 0
$$

and the equality holds if and only if $f_{\lambda}$ has no singular fibers. Now if $\chi\left(\mathcal{O}_{S_{\lambda}}\right)=0$, then $h$ is an elliptic fiber bundle. If $\chi\left(\mathcal{O}_{S_{\lambda}}\right)=1$, then $q\left(S_{\lambda}\right)=0$, and hence $S_{\lambda}$ is either a rational surface or an Enriques surface. The latter case does not occur, since Enriques surfaces have no fibrations with a section. Finally we exclude the case when $S_{\lambda}$ is a rational surface. We may distinguish between two cases:

1) $q(S)=1$. If $f: S \rightarrow C$ has constant moduli, then $f$ is the (relatively minimal) smooth model of $F \times \tilde{C} / \tau_{F} \times \tau_{\tilde{C}} \rightarrow \tilde{C} / \tau_{\tilde{C}}$, where $\tau_{F}$ is a bi-elliptic involution of $F$ and $\tau_{\tilde{C}}$ is the hyperelliptic involution of $\tilde{C}$. Hence $f$ has singular fibers of type $\left(b_{0}\right)$ of 2.6. This is a contradiction by Lemma 4.7(ii). Now we assume that $f: S \rightarrow C$ has variable moduli. Consider the morphism $\psi=f \times$ alb : $S \rightarrow C \times \operatorname{alb}(S)$, where 
alb: $S \rightarrow \operatorname{alb}(S)$ is the Albanese map. If $\operatorname{deg} \psi=2$, let $\eta$ be the involution of $S$ corresponding to $\psi$, then $\eta \circ \tau$ induces trivial action on $H^{0}\left(S, \omega_{S}\right)$. By the proof of Theorem 3.2(i), $\eta \circ \tau=\sigma$. This implies $\eta=\lambda$, and hence $C \times \operatorname{alb}(S)$ is birationally isomorphic to $S_{\lambda}$, a contradiction. If $\operatorname{deg} \psi \geq 3$, then each singular fiber of $f$ is semistable [X1, p. 47]. So by Lemma 4.6] and [H] Theorem 3], $K_{S}^{2}=2 \chi\left(\mathcal{O}_{S}\right)-6$, and hence $q(S)=0$, a contradiction.

2) $q(S)=0$. Notations as in 2.4 We show that, if $D_{\lambda}$ is a section of $f_{\lambda}$, let $\tilde{D}_{\lambda}$ be the strict transform of $D_{\lambda}$ in $\tilde{S} / \tilde{\lambda}, \tilde{D}=\pi^{*} \tilde{D}_{\lambda}$ and $D=\rho(\tilde{D})$, then either $D=H$ or $D$ is $\tau$-fixed. In particular, $f_{\lambda}$ has at most finite many sections, and hence the rank of the Mordell-Weil group of the generic fiber of $f_{\lambda}$ is zero. Otherwise, we have $\sigma(D) \neq D$. If there is a $\lambda$-isolated fixed point $p \in D$, then both $p$ and $\sigma(p)$ are singular points of $F^{\prime}=f^{*} f(p)$. By the classification of singular fibers of $f$ and the action of $\langle\tau, \sigma\rangle$ on them (Lemmas 4.7, 4.9, 4.10), we have $\sigma(p)=p$. This implies

$$
\rho^{*}(D-\sigma(D)) \equiv \tilde{D}-\widetilde{\sigma(D)} \equiv \pi^{*}\left(\tilde{D}_{\lambda}-\pi(\widetilde{\sigma(D)})\right),
$$

where $\widetilde{\sigma(D)}$ is the strict transform of $\sigma(D)$ in $\tilde{S}$. Since $\sigma$ acts trivially on $H^{2}(S, \mathbb{Q})$ and $q(S)=0$, by the exact sequence of the exponential, we have that either $D \equiv$ $\sigma(D)$ or $D-\sigma(D)$ is a torsion element in $H^{2}(S, \mathbb{Z})$. Since $H^{2}(\tilde{S} / \tilde{\lambda}, \mathbb{Z})$ is torsion free, by (4.12.1), the second case does not occur. Now $D \equiv \sigma(D)$ implies $h^{0}\left(D_{\mid F}\right)=2$, where $F$ is a general fiber of $f$. So $D$ is $\tau$-invariant. Since $D$ is $\lambda$-invariant, we get $D$ is $\sigma$-invariant, a contradiction.

Now we have

$$
\begin{aligned}
2+\sum_{\text {singular fiber } F_{\lambda}^{\prime}}\left(n_{F_{\lambda}^{\prime}}-1\right) & =\operatorname{rank} N S\left(S_{\lambda}\right)=10, \\
\sum_{\text {singular fiber } F_{\lambda}^{\prime}} e\left(F_{\lambda}^{\prime}\right) & =e\left(S_{\lambda}\right)=12,
\end{aligned}
$$

where $n_{F_{\lambda}^{\prime}}$ is the number of irreducible components of a singular fiber $F_{\lambda}^{\prime}$. By Corollaries 4.8 and 4.11 , the type of each fiber of $f_{\lambda}$ is $\mathrm{I}_{0}, \mathrm{I}_{1}, \mathrm{I}_{2}$, II or III.

\begin{tabular}{|l|l|l|l|l|}
\hline$F_{\lambda}^{\prime}$ & $\mathrm{I}_{1}$ & $\mathrm{I}_{2}$ & $\mathrm{II}$ & $\mathrm{III}$ \\
\hline$n_{F_{\lambda}^{\prime}}-1$ & 0 & 1 & 0 & 1 \\
\hline$e\left(F_{\lambda}^{\prime}\right)$ & 1 & 2 & 2 & 3 \\
\hline
\end{tabular}

Using the table above, we easily see that there are no possible configurations of degenerate fibers on $S_{\lambda}$ satisfying 4.12 .2 .

Proof of (ii) of Theorem 3.2. By Proposition 4.12, $f_{\lambda}: S_{\lambda} \rightarrow C$ is an elliptic fiber bundle. So by Corollaries 4.8 and 4.11, $f_{\mid H}: H \rightarrow C$ is étale, and the type of each singular fiber $F^{\prime}$ and on which the action of $\sigma$ is as in (i), (v) of Lemma 4.9, or (ii) of Lemma 4.7 with $\Gamma$ smooth.

\begin{tabular}{|l|l|l|l|}
\hline$F^{\prime}$ & (i) of 4.9 & (v) of 4.9 & (ii) of 4.7 \\
\hline Isolated $\sigma$-fixed points on $F^{\prime}$ & 1 & $\mathrm{n}+1$ & 3 \\
\hline$\sigma$-fixed curves on $F^{\prime}$ & 0 & 0 & $(\mathrm{k}+1) / 2$ \\
\hline$e_{F^{\prime}}=e\left(F^{\prime}\right)+2$ & 1 & $\mathrm{n}+1$ & $\mathrm{k}+4$ \\
\hline Horikawa number $H\left(F^{\prime}\right)$ & 0 & 0 & $\mathrm{k}+1$ \\
\hline
\end{tabular}

Let $x$ be the number of fibers of type (i) of Lemma 4.9. For $n \geq 1$, let $y_{n}$ be the number of fibers $F^{\prime}=\Gamma+\Theta$ as in (v) of Lemma 4.9, with $\Theta$ a -2-cycle of type $A_{n}$. 
For odd $k \geq 1$, let $z_{k}$ be the number of fibers $F^{\prime}=\Theta+\Theta^{\prime}+2 \Theta_{1}+\cdots+2 \Theta_{k}+2 \Gamma$ as in (ii) of Lemma 4.7, with $\Gamma$ smooth. By the above table,

$$
\begin{array}{r}
e\left(S^{\sigma}\right)=x+\sum_{n \geq 1}(n+1) y_{n}+\sum_{\text {odd } k \geq 1}(k+4) z_{k}+e(H), \\
e(S)-4(g(C)-1)=x+\sum_{n \geq 1}(n+1) y_{n}+\sum_{\text {odd } k \geq 1}(k+4) z_{k} .
\end{array}
$$

Note that

$$
e(H)=-\left(K_{S} H+H^{2}\right)= \begin{cases}0 & \text { if } g(C)=1, \\ 4 & \text { if } g(C)=0,\end{cases}
$$

by (4.12.3) and the topological Lefschetz formula (cf. [AS, p. 566]), we have

$$
q(S)-\operatorname{dim} H^{0}\left(S, \Omega_{S}^{1}\right)^{\sigma}= \begin{cases}0 & \text { if } g(C)=1, \\ 1 & \text { if } g(C)=0 .\end{cases}
$$

We have

$$
K_{S} \equiv M+H+\sum_{\text {singular fiber } F^{\prime}} \Delta_{F^{\prime}}
$$

where $\Delta_{F^{\prime}}$ is as in 2.9. So

$$
K_{S} H=2 d+H^{2}+\sum_{\text {odd } k \geq 1}(k+1) z_{k},
$$

where $d$ is as in (3.2.2). By (4.12.4) and (4.12.6), we get

$$
\begin{aligned}
K_{S}^{2} & =K_{S} M+K_{S} H+\sum_{\text {singular fiber } F^{\prime}} K_{S} \Delta_{F^{\prime}} \\
& =3 d+\sum_{\text {odd } k \geq 1} \frac{(k+1)}{2} z_{k}+ \begin{cases}0 & \text { if } g(C)=1, \\
-2 & \text { if } g(C)=0 .\end{cases}
\end{aligned}
$$

By [H], Theorem 3],

$$
K_{S}^{2}-2 \chi\left(\mathcal{O}_{S}\right)-6(g(C)-1)=\sum_{\text {odd } k \geq 1}(k+1) z_{k} .
$$

Combining (4.12.7), (4.12.8) and (4.12.5), we finish the proof of Theorem 3.2(ii).

\section{ACKNOWLEDGMENTS}

The author thanks the referee for a careful reading of the paper and some useful suggestions. This work has been supported by the NSFC (No. 10271005) and SRF for ROCS, SEM.

\section{REFERENCES}

[AS] M. Atiyah, I. M. Singer, The index of elliptic operators III, Ann. of Math. 87 (1968) 546-604. MR0236952 (38:5245)

[Be] A. Beauville, L'application canonique pour les surfaces de type qénéral, Invent. Math. 55 (1979) 121-140. MR0553705 (81m:14025)

[BPV] W. Barth, C. Peters and A. Van de Ven, Compact complex surfaces, Ergeb. Math. Grenzgeb. (3), Springer-Verlag, Berlin, 1984. MR0749574 (86c:32026)

[BR] D. Burns and M. Rapoport, On the Torelli problem for Kählerian K3 surfaces, Ann. Sci. École Norm. Sup. 4 (1975) 235-274. MR0447635 (56:5945) 
[Ca1] J.-X. Cai, On Abelian automorphism groups of fiber surfaces of small genus, Math. Proc. Cambridge Philos. Soc. 130 (2001) 161-174. MR.1797736 (2002a:14049)

[Ca2] J.-X. Cai, Automorphisms of a surface of general type acting trivially in cohomology, Tohoku Math. J. (2) 56 (2004), 341-355. MR2075770

$[\mathrm{H}]$ E. Horikawa, On algebraic surfaces with pencils of curves of genus 2, in Complex analysis and algebraic geometry, a volume dedicated to Kodaira, Cambridge 1977, 79-90. MR0453756 (56:12015)

[Mu] D. Mumford, Algebraic Geometry I: Complex Projective Varieties, Springer-Verlag, 1976. MR0453732 (56:11992)

[Per] U. Persson, Double coverings and surfaces of general type, in Algebraic geometry, LNM 687 (1978) 168-195. MR0527234 (80h:14017)

[Pet] C. A. M. Peters, Holomorphic automorphisms of compact Kahler surfaces and their induced actions in cohomology, Invent. Math. 52 (1979), 143-148. MR.0536077 (80h:32050)

[Ue] K. Ueno, A remark on automorphisms of Enriques surfaces, J. Fac. Sci. Univ. Tokyo, Sect. I A Math. 23 (1976) 149-165. MR0404268 (53:8071)

[X1] G. Xiao, Surfaces fibrees en courbes de genre deux, LNM 1137, Springer-Verlag, 1985. MR0872271 (88a:14042)

[X2] G. Xiao, L'irrégularité des surfaces de type général dont le système canonique est composé d'un pinceau, Compositio Math. 56 (1985) 251-257. MR0809870 (87d:14031)

LMam, School of Mathematical Sciences, Peking University, Beijing 100871, PeoPLE'S REPUBLIC OF CHINA

E-mail address: cai@math.pku.edu.cn 